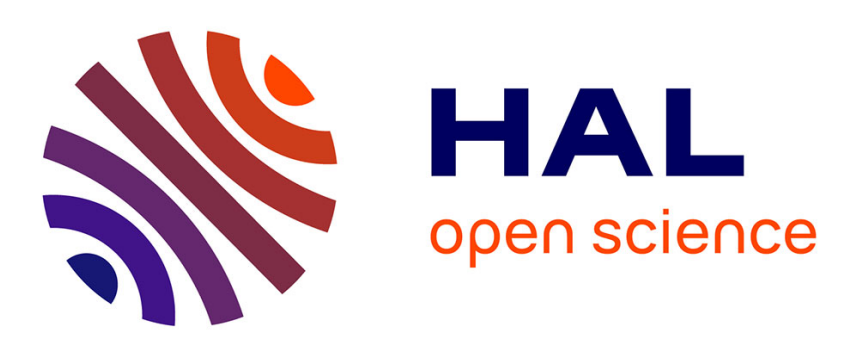

\title{
Accumulation des métaux lourds dans Eisenia fetida andrei (Oligochaeta, Lumbricidae) élevé sur ordures ménagères
}

Monique Linères, Léon Fayolle, Jean Tauzin, Christian Juste

\section{- To cite this version:}

Monique Linères, Léon Fayolle, Jean Tauzin, Christian Juste. Accumulation des métaux lourds dans Eisenia fetida andrei (Oligochaeta, Lumbricidae) élevé sur ordures ménagères. Agronomie, 1985, 5 (9), pp.779-784. hal-00884811

\section{HAL Id: hal-00884811 https://hal.science/hal-00884811}

Submitted on 1 Jan 1985

HAL is a multi-disciplinary open access archive for the deposit and dissemination of scientific research documents, whether they are published or not. The documents may come from teaching and research institutions in France or abroad, or from public or private research centers.
L'archive ouverte pluridisciplinaire HAL, est destinée au dépôt et à la diffusion de documents scientifiques de niveau recherche, publiés ou non, émanant des établissements d'enseignement et de recherche français ou étrangers, des laboratoires publics ou privés. 


\title{
Accumulation des métaux lourds dans Eisenia fetida andrei (Oligochaeta, Lumbricidae) élevé sur ordures ménagères
}

\author{
Monique LINÈRES, Léon FAYOLLE (*), Jean TAUZIN et Christian JUSTE \\ I.N.R.A., Station d'Agronomie, Centre de Recherches de Bordeaux, B.P. 131, 33140 Pont-de-la-Maye \\ (*) Station de Recherches sur la Faune du Sol, BV 1540, F 21034 Dijon Cedex
}

\begin{abstract}
On a déterminé les teneurs en $\mathrm{Cd}, \mathrm{Cr}, \mathrm{Ni}, \mathrm{Pb}$ et $\mathrm{Zn}$ des vers de terreau, Eisenia fetida andrei, élevés dans un compost d'ordures ménagères âgé de 7 jours et dans un compost de référence (à base de terreau et de fèces de lapin). L'élevage a été réalisé à $25^{\circ} \mathrm{C}$, en containers de $5 \mathrm{~kg}$ de compost humide ensemencés avec 200 cocons pour une durée de 10 semaines. La production de biomasse lombricienne et le poids moyen des vers sont très significativement augmentés sur ordures ménagères. Seule la teneur en $\mathrm{Pb}$ et $\mathrm{Zn}$ des vers augmente quand ces derniers sont élevés dans les ordures ménagères, alors que les variations des teneurs en $\mathrm{Cd}$, $\mathrm{Cr}$ et $\mathrm{Ni}$, quand on change de milieu d'élevage, demeurent non significatives en raison d'une pollution initiale du compost de référence par ces métaux.

L'élimination du contenu du tube digestif par un séjour de $24 \mathrm{~h}$ sur papier filtre entraîne une baisse importante des teneurs en $\mathrm{Cr}, \mathrm{Pb}, \mathrm{Ni}$ et $\mathrm{Zn}$ d'E. $f$. andrei élevé dans les ordures ménagères mais est sans action sur la teneur en $\mathrm{Cd}$, métal qui doit donc être considéré comme très fortement lié au lombricien.
\end{abstract}

Mots clés additionnels : Lombricompostage, biomasse lombricienne, $\mathrm{Cd}, \mathrm{Cr}, \mathrm{Ni}, \mathrm{Pb}, \mathrm{Zn}$, tube digestif, jeûne, Annelida.

\begin{abstract}
Accumulation of heavy metals in Eisenia fetida andrei (Oligochaeta, Lumbricidae) grown in garbage.

The weight gain of a worm commonly found in manure piles (Eisenia fetida andrei) was measured after a 10 week-period of growth in fresh garbage (7 day-old) or in control compost (rabbit faeces - table 2) put in plastic boxes arranged in a growth chamber at $25^{\circ} \mathrm{C}$. The effect of the medium on $\mathrm{Cd}, \mathrm{Cr}, \mathrm{Ni}, \mathrm{Pb}$ and $\mathrm{Zn}$ accumulation in worms, before or after discharge of excreta for $24 \mathrm{~h}$ in Petri dishes containing pieces of wet filter, was also measured. Worm biomass production and individual weights were more significantly increased in garbage than in control compost (table 3 ). $\mathrm{Pb}$ and $\mathrm{Zn}$ accumulated in worms growing in garbage, whereas $\mathrm{Cd}, \mathrm{Cr}$ and $\mathrm{Ni}$ did so no more than in control compost because this latter was polluted by the metals (table 4). Discharge of faeces and excreta induced a strong decrease of $\mathrm{Cr}, \mathrm{Ni}, \mathrm{Pb}$ and $\mathrm{Zn}$ concentration in $E$. $f$. andrei growing in garbage but no effect of this treatment was observed for $\mathrm{Cd}$, this later being strongly bound in worm tissues (table 5).
\end{abstract}

Additional key words : Earthworm composting, earthworm biomass, $\mathrm{Cd}, \mathrm{Cr}, \mathrm{Ni}, \mathrm{Pb}, \mathrm{Zn}$, intestinal content, fast, Annelida.

\section{INTRODUCTION}

L'étude des possibilités de valorisation de certains déchets organiques par leur utilisation comme support d'élevage d'Eisenia fetida (SAvignY, 1826) (Vermicompostage, compélevage, lombricompostage...) a fait depuis quelques années l'objet d'un nombre relativement important de travaux parmi lesquels on peut citer ceux de FRANZ (1978), MITCHELL (1978), NEUHAUSER (1978), CARMODY (1979), MitCheLl et al.
(1980), SABine (1980), APPElhof (1981), FAyOlle (1982). Très schématiquement, l'intérêt du développement d'une telle technologie résiderait : d'une part, dans une accélération du processus de dégradation et de stabilisation des matériaux organiques valorisables comme amendements et, d'autre part, dans la production de vers utilisables, en raison de leur richesse en protéines, comme aliment pour les animaux d'élevage (porcs, poulets, poissons).

La réalisation de cet objectif suppose que soient 
parfaitement définis les paramètres de développement optimal des vers dans ces milieux très particuliers et que la qualité des lombrics obtenus soit compatible avec les normes de composition auxquelles doivent satisfaire les aliments. A cet égard, certains des déchets dont l'emploi peut être envisagé à de telles fins (boues de station d'épuration, ordures ménagères) se caractérisent souvent par des richesses importantes en métaux lourds. La possibilité de transfert de ces derniers dans $E$. fetida constitue donc un risque à évaluer dans la mesure où il a déjà été démontré que diverses espèces de vers qui vivent dans des sols pollués par des métaux en raison de la proximité d'autoroutes (GISH \& CHRISTENSEN, 1973), d'exploitations minières (IRELAND, 1975) ou de l'épandage intentionnel de déchets organiques (HELMKE et al., 1979) ont des teneurs en métaux lourds, et notamment en Cd et $\mathrm{Zn}$, beaucoup plus élevées que ceux qui se développent en zone non polluée. IRELAND (1983) et surtout HARTENSTEIN et al. (1980) ont contribué à clarifier les concepts d'accumulation et de concentration des métaux lourds : il y a accumulation, soit quand on assiste à une augmentation progressive de la concentration du métal dans l'animal au cours du temps, soit quand cette concentration s'élève parallèlement à celle du milieu d'élevage ; selon ces mêmes auteurs, il n'y a véritablement concentration que lorsque le rapport de la richesse métallique dans l'organisme à celle du milieu est supérieur à l'unité. Dans le cas particulier d'E. fetida, la notion d'accumulation et/ou de concentration est rendue complexe par l'existence d'un contenu intestinal important dont la composition peut se rapprocher d'autant plus de celle du milieu d'élevage que celui-ci est plus homogène ; une meilleure approche de la connaissance de l'accumulation réelle suppose donc l'élimination préalable de ce contenu. Il n'en reste pas moins que l'accumulation d'un métal donné dépend fortement de la nature des milieux d'élevage (essentiellement, teneur et nature de la matière organique, addition ou non de métaux...). La majorité des recherches réalisées conduisent cependant à reconnaître que $\mathrm{Zn}$ et surtout $\mathrm{Cd}$ sont les métaux qui donnent lieu à l'accumulation la plus marquée, des indices de concentration de 2 à 7,5 pouvant être observés pour ce dernier métal dans des vers se développant dans certains composts (MORI \& KURIHARA, 1979). Par contre, pour d'autres métaux tels que le sélénium, le chrome et le mercure, il peut y avoir ou non accumulation, voire concentration, selon la nature du substrat d'élevage (BEYER, 1980).

S'il existe un nombre important de résultats concernant le lombricompostage sur des matières organiques conventionnelles telles que fumier, divers déchets de l'agroalimentaire et boues de station d'épuration, les recherches concernant la valorisation des ordures ménagères par cette technologie sont assez peu nombreuses (GRAFF, 1974 ; FAYOLLE, 1982 ; de BLIGNERES, 1983 ; BOUCHÉ, 1983), ce qui peut paraître surprenant compte tenu du volume important de déchets de cette nature produit chaque année. Le caractère hétérogène, la pauvreté relative en azote, la présence d'une quantité importantes d'inertes « a priori " non utilisables par les lombriciens expliquent en partie ce désintéressement. Il faut y ajouter aussi une charge non négligeable en métaux lourds divers (tabl. 1) qui

\section{TABLEAU 1}

Comparaison des teneurs moyennes en métaux lourds d'un compost d'ordures ménagères et d'une boue de station d'épuration urbaine (en $\mathrm{mg} / \mathrm{kg}$ de matière sèche) (C. JUSTE et al., 1979).

Average values of heavy metal content in garbage and sewage sludge.

\begin{tabular}{cccc}
\hline Eléments & $\begin{array}{c}\text { Compost d'ordures ménagères } \\
(120 \text { jours de compostage })\end{array}$ & $\begin{array}{c}\text { Boue de station } \\
\text { d'épuration urbaine }\end{array}$ \\
\hline $\mathrm{Cu}$ & $357 \pm 135^{*}$ & 850 \\
$\mathrm{Mn}$ & $854 \pm 105^{*}$ & 260 \\
$\mathrm{Fe}$ & $17110 \pm 3250^{*}$ & 11000 \\
$\mathrm{Zn}$ & $1525 \pm 235^{*}$ & 1740 \\
$\mathrm{~Pb}$ & $599 \pm 180^{*}$ & 500 \\
$\mathrm{Cd}$ & $8,5 \pm 1,5^{*}$ & 16 \\
$\mathrm{Hg}$ & $3,4 \pm$ & $1,3^{*}$ & 5 \\
$\mathrm{Cr}$ & $219 \pm 35^{*}$ & 82 \\
$\mathrm{Ni}$ & $196 \pm 25^{*}$ & \\
\end{tabular}

(*) Intervalle de confiance au seuil de $5 \%$ calculé à partir de résultats d'analyse de 33 échantillons d'origines géographiques différentes.

représente un risque d'altération de la qualité des vers produits sur ces substrats.

L'objet du présent travail est d'évaluer l'importance de ce risque, en comparant les possibilités d'accumulation réelle de $\mathrm{Cd}, \mathrm{Pb}, \mathrm{Cr}$, Ni et $\mathrm{Zn}$ dans $E$. fetida élevé sur un terreau de référence à faible charge métallique, d'une part, et sur ordures ménagères frâ̂ches, d'autre part.

\section{MATÉRIEL ET MÉTHODES}

\section{A. Elevage des vers}

Il a été conduit sur des ordures ménagères de la ville de Montargis (Loiret) qui ont subi une fermentation de 7 jours (procédé TRIGA) puis un criblage sur tamis à mailles de $30 \mathrm{~mm}$. On a employé, comme milieu d'élevage de référence, un mélange contenant, en poids sec, $1 / 3$ de fèces de lapin (animaux nourris avec des granulés du commerce) et $2 / 3$ d'un terreau commercial à base de tourbe brune d'Arendon (Ain). Les 2 milieux, dont la composition figure au tableau 2 , ont subi une évolution préalable de $15 \mathrm{j}$ en couche mince. Les vers utilisés appartiennent à une souche locale de l'espèce $E$. fetida andrei (BOUCHÉ, 1972).

Les matières organiques à 60 p. 100 d'humidité ( $5 \mathrm{~kg}$ en poids frais) sont disposées dans des pots circulaires (diamètre $175 \mathrm{~mm}$, hauteur $550 \mathrm{~mm}$ ) munis de tissu à maille fine à la base et au sommet pour faciliter l'aération. Chaque pot est ensemencé avec 200 cocons âgés de 0 à 3 semaines. Les élevages sont réalisés en enceinte climatisée à $25^{\circ} \mathrm{C}$, l'humidité étant voisine de la saturation.

Après 10 semaines d'évolution, les vers et cocons sont récupérés par simple triage manuel.

\section{B. Protocole expérimental}

L'essai comporte au total 10 pots contenant des ordures ménagères et 5 pots contenant le milieu d'élevage de référence. A l'issue des 10 semaines, les vers 
TABLEAU 2

Caractéristiques initiales des substrats d'élevage utilisés. Characteristics of the growth media.

\begin{tabular}{|c|c|c|}
\hline & $\begin{array}{l}\text { Ordures } \\
\text { ménagères }\end{array}$ & $\begin{array}{c}\text { Substrat } \\
\text { de référence } \\
\left({ }^{*}\right)\end{array}$ \\
\hline $\mathrm{pH} \ldots \ldots \ldots \ldots \ldots$ & 7,6 & 8,2 \\
\hline Résistivité $\left(\Omega \cdot \mathrm{cm}^{2} \cdot \mathrm{cm}^{1}{ }^{1}\right)$ & 375 & 384 \\
\hline Cendres $\ldots . .(\%$ M.S. $)$ & 28,1 & 53,3 \\
\hline Matière organique ... & 71,9 & 46,7 \\
\hline Carbone ........ & 35,7 & 24,4 \\
\hline Azote $\ldots \ldots \ldots \ldots$ & 1,06 & 1,30 \\
\hline Rapport C/N ..... & 33,7 & 18,8 \\
\hline $\mathrm{Zn} \ldots \ldots(\mathrm{mg} / \mathrm{kg}$ M.S. $)$ & 1105 & 112 \\
\hline $\mathrm{Pb} \ldots \ldots \ldots \ldots$ & 136 & 4 \\
\hline $\mathrm{Cd} \ldots \ldots \ldots \ldots$ & 2,8 & 0,2 \\
\hline $\mathrm{Cr} \ldots \ldots \ldots \ldots$ & 131 & 70 \\
\hline $\mathrm{Ni} \ldots \ldots \ldots \ldots$ & 68 & 76 \\
\hline
\end{tabular}

(tous stades confondus) provenant de 5 pots avec ordures et 5 pots témoins sont immédiatement lavés, pesés, congelés puis lyophilisés tube digestif plein. Les vers issus des 5 derniers pots contenant les ordures ménagères subissent un traitement particulier : après lavage et pesée, ils sont placés pendant $24 \mathrm{~h}$ à $25^{\circ} \mathrm{C}$ sur un papier filtre humide renouvelé 2 fois, sur lequel ils abandonnent fèces, mucus et urine. Une période de 24 h (MORI \& KURIHARA, 1979) avec renouvellement du papier filtre pour éviter une réingestion des fèces (TARRADELAS et al., 1982) paraît suffisante pour remplacer le milieu d'élevage du tube digestif de l'animal, HARTENSTEIN et al. (1981) ayant montré que E. fetida andrei a un transit intestinal d'une durée de $2 \mathrm{~h}$ et demie environ lorsqu'il est maintenu à $25^{\circ} \mathrm{C}$. Le milieu de purge (papier filtre + déjections) est séché, pesé, puis conservé pour analyse, de manière à permettre la comparaison de sa composition avec celle des "turricules" récupérés par tri manuel au sein des milieux d'élevage.

\section{Déterminations réalisées}

A la $10^{\mathrm{e}}$ semaine, on note l'état de la population lombricienne de la $1^{\text {re }}$ génération : classes d'âge et poids des animaux.

Les vers lyophilisés et les turricules sont minéralisés selon la technique décrite par HOENIG et al. (1982) : les échantillons non broyés sont minéralisés au four électrique avec montée progressive de la température jusqu'à $450^{\circ}$, cette dernière étant maintenue pendant $16 \mathrm{~h}$. Après refroidissement, on ajoute aux cendres un mélange $(50 / 50)$ d'acide nitrique concentré (R.P. suprapur) et une solution à 30 p. 100 de péroxyde d'hydrogène ("Perhydrol » R.P. suprapur), mélange qui est porté à ébullition pendant $15 \mathrm{mn}$. Après refroidissement et filtration, les métaux sont dosés par spectrophotométrie d'absorption atomique à flamme (Varian-Techtron 1475). Les mélanges de papier filtre et de déjections et le papier filtre seul sont minéralisés et analysés selon la même technique, mais après broyage au mixer.
Les ordures ménagères et le milieu d'élevage de référence sont séchés à l'air, broyés dans un broyeur Gondard. Les métaux sont dosés également par absorption atomique après minéralisation par voie sèche et reprise des cendres par l'acide chlorhydrique pur.

La détermination de la concentration en métaux dans le mélange papier filtre et déjections, d'une part, et, d'autre part, dans le papier filtre témoin qui a servi de support de purge a permis de calculer les concentrations en métaux des diverses déjections de l'animal.

\section{RÉSULTATS}

\section{A. Production de vers (tabl. 3)}

Le nombre de vers récolté après 10 semaines ne diffère pas de façon significative entre les 2 supports d'élevage ; un ensemencement avec 200 cocons a permis d'obtenir 266 individus sur ordures ménagères et 320 sur substrat de référence. La biomasse lombricienne est plus élevée sur ordures ménagères; cette supériorité des déchets urbains est encore plus nette si l'on considère le poids moyen individuel des vers. Par contre, la production de cocons est identique pour les 2 milieux, malgré un pourcentage plus élevé d'adultes sur ordures ménagères.

\section{B. Accumulation de métaux dans $E$. $f$. andrei élevé pendant 10 semaines sur ordures ménagères (tabl. 4)}

Si l'on s'en tient à la définition donnée par HARTENSTEIN et al. (1980), il n'y a accumulation que dans le cas du zinc et du plomb. La richesse relative en $\mathrm{Ni}$ et $\mathrm{Cr}$ et, dans une moindre mesure, en $\mathrm{Cd}$ du substrat de référence explique que, dans le cas de cette expérience, il n'y a pas à proprement parler d'" accumulation " de ces 3 métaux dans les vers issus des ordures ménagères. La variation non significative de la teneur en $\mathrm{Cd}$ des vers, alors que la richesse en ce métal du

\section{TABLEAU 3}

Production de biomasse lombricienne après 10 semaines d'élevage à $25^{\circ} \mathrm{C}$

Worm biomass production through a growth period of 10 weeks at $25^{\circ} \mathrm{C}$.

\begin{tabular}{lccc}
\hline \hline & $\begin{array}{c}\text { Ordures } \\
\text { ménagères }\end{array}$ & $\begin{array}{c}\text { Substrat } \\
\text { de } \\
\text { référence }\end{array}$ & $\begin{array}{c}\text { Degré de } \\
\text { signification } \\
\text { de la } \\
\text { différence }\end{array}$ \\
\hline $\begin{array}{l}\text { Nombre de vers par } \\
\text { cocon mis en élevage }\end{array}$ & 1,33 & 1,60 & N.S. \\
$\begin{array}{l}\text { Poids frais des vers } \\
\text { par pot (en g) }\end{array}$ & 93,9 & 67,0 & S. \\
$\begin{array}{l}\text { Poids moyen indivi- } \\
\text { duel (mg) }\end{array}$ & 374,9 & 214,5 & H.S. \\
$\begin{array}{l}\text { Pourcentage d'adultes } \\
\text { Nombre de cocons } \\
\text { pondus par les nou- } \\
\text { veaux géniteurs }\end{array}$ & 74,9 & 6,0 & T.H.S. \\
\hline \hline
\end{tabular}


TABLEAU 4

Enrichissement en métaux lourds d'Eisenia fetida andrei en fonction de la nature du milieu d'élevage (mg/kg de matière sèche). Relationship between heavy metal accumulation in Eisenia fetida andrei and the nature of the growth medium (ppm dry matter levels).

\begin{tabular}{|c|c|c|c|c|c|c|}
\hline & Nature du milieu & $\mathrm{Zn}$ & $\mathrm{Cd}$ & $\mathrm{Ni}$ & $\mathrm{Pb}$ & $\mathrm{Cr}$ \\
\hline $\begin{array}{c}\text { Milieu } \\
\text { d'élevage }\end{array}$ & $\begin{array}{l}\text { Ordures ménagères } \\
\text { Substrat de référence (Terreau }+ \text { fèces de } \\
\text { lapin) }\end{array}$ & $\begin{array}{l}1270 \pm 110\left(^{* *}\right) \\
165 \pm 9\end{array}$ & $\begin{array}{l}5,02 \pm 0,94 \\
0,46 \pm 0,13\end{array}$ & $\begin{array}{r}135 \pm 34 \\
69 \pm 13\end{array}$ & $\begin{array}{r}227 \pm 112 \\
6,8 \pm 1,2\end{array}$ & $\begin{array}{r}159 \pm 11 \\
65 \pm 12\end{array}$ \\
\hline $\begin{array}{l}\text { Eisenia } \\
\text { fetida } \\
\text { andrei }\end{array}$ & $\begin{array}{l}\text { Ordures ménagères } \\
\text { Substrat de référence } \\
\text { Degré de signification de la différence }\end{array}$ & $\begin{array}{c}358 \\
134 \\
\text { T.H.S. }\end{array}$ & $\begin{array}{r}6,0 \\
5,2 \\
\text { N.S. }\end{array}$ & $\begin{array}{r}11,0 \\
8,6 \\
\text { N.S. }\end{array}$ & $\begin{array}{r}49,3 \\
3,0 \\
\text { T.H.S. }\end{array}$ & $\begin{array}{l}37,2 \\
33,0 \\
\text { N.S. }\end{array}$ \\
\hline $\begin{array}{c}\text { Indice }\left(^{*}\right) \\
\text { d'accumulation } \\
\text { moyen }\end{array}$ & $\begin{array}{l}\text { Ordures ménagères } \\
\text { Substrat de référence } \\
\text { Degré de signification de la différence }\end{array}$ & $\begin{array}{c}0,29 \\
0,81 \\
\text { T.H.S. }\end{array}$ & $\begin{array}{c}0,96 \\
11,5 \\
\text { T.H.S. }\end{array}$ & $\begin{array}{l}0,08 \\
0,13 \\
\mathrm{~S} .\end{array}$ & $\begin{array}{c}0,22 \\
0,39 \\
\text { N.S. }\end{array}$ & $\begin{array}{r}0,23 \\
0,55 \\
\text { T.H.S. }\end{array}$ \\
\hline
\end{tabular}

${ }^{*}$ ) Indice d'accumulation $=$ concentration du métal dans $E$. fetida/concentration du métal dans le milieu d'élevage (**) Intervalle de confiance au seuil de $5 \%$.

milieu d'élévage est décuplée, pourrait avoir pour origine, soit l'ingestion préférentielle par les vers élevés sur ordures d'aliments moins chargés en $\mathrm{Cd}$, soit l'ingestion de matières chargées en $\mathrm{Cd}$ dans le cas des lombriciens élevés sur terreau de référence. A l'opposé, l'accumulation de $\mathrm{Zn}$ et de $\mathrm{Pb}$ correspondrait à l'ingestion de matériaux plus riches en ces éléments par les vers élevés sur ordures ménagères.

L'examen des indices d'accumulation permet de bien faire apparaître l'aptitude spécifique de certains métaux à s'accumuler préférentiellement et cela d'autant plus que le substrat d'élevage en renferme moins. Dans le cas présent, le cadmium se singularise par une très forte tendance à la concentration (indice sur terreau témoin $=11,5$ ), confirmant ainsi les observations faites par de très nombreux auteurs.

\section{Influence d'un jeûne de 24 h sur la teneur en métaux lourds d' $E$. $f$. andrei}

Les pesées de lombriciens effectuées avant et après jeûne montrent que ces derniers subissent au cours de ce traitement de $24 \mathrm{~h}$ une perte pondérale de matière sèche représentant 20 à 25 p. 100 du poids initial (compte tenu du poids de papier filtre ingéré). Cette perte, qui correspond aux fèces, mucus et urine, modifie sensiblement la teneur en certains métaux lourds (tabl. 4). On constate en effet que les richesses en $\mathrm{Zn}, \mathrm{Ni}, \mathrm{Pb}$ et $\mathrm{Cr}$ sont très significativement abaissées ; par contre, la teneur en $\mathrm{Cd}$ ne varie pas. Il en résulte que les indices d'accumulation sont eux-mêmes affectés de manière significative à l'exception de celui correspondant au cadmium.

\section{Comparaison de la composition des ordures ména- gères, des " turricules" et des déjections obtenues au cours du jeûne (tabl. 5)}

On observe que la composition des «turricules» prélevés par tri manuel au sein du milieu d'élevage est très sensiblement analogue à celle des déjections produites lors du jeûne. On peut donc dire que le produit obtenu « in vitro » au cours de cette opération constitue un bon reflet des déjections éliminées " in situ » lors de l'élevage.

La comparaison des teneurs en métaux lourds de l'ensemble des déjections et de celles caractérisant les ordures ménagères fait apparaître qu'il existe une différenciation marquée, très dépendante de la nature du métal : il y a en effet, par rapport au milieu d'élevage, enrichissement des déjections en $\mathrm{Zn}$, appauvrissement en $\mathrm{Cr}$ et $\mathrm{Ni}$ et pas de variation significative en $\mathrm{Cd}$ et $\mathrm{Pb}$. Cette différenciation repose très certainement sur le fait que les lombriciens qui se développent dans le milieu très hétérogène que constituent des ordures ménagères procèdent à un certain tri des matières qu'ils consomment et dont la teneur en métaux lourds peut varier en fonction de leur nature. Cela ne parait pas être le cas pour le cadmium dont la concentration dans les déjections est peu modifiée : il semble donc que les substances ingérées par les vers ont sensiblement la même charge en ce métal, ce qui infirmerait l'une des deux hypothèses émises précédemment (ingestion préférentielle de matériaux peu chargés en Cd sur ordures ménagères) mais confirmerait l'autre (ingestion préférentielle de matières très chargées en $\mathrm{Cd}$ sur terreau de référence).

\section{DISCUSSION ET CONCLUSION}

Les ordures ménagères constituent un milieu très favorable pour la croissance d'E. fetida, la production de $18,8 \mathrm{~g}$ de vers frais par $\mathrm{kg}$ de déchet urbain humide est supérieure à celle obtenue sur un milieu à base de fèces de lapin (soit 13,4 g). Si l'on considère une période plus courte (4 à 6 semaines) la croissance des vers est plus rapide sur excréments animaux que sur ordures ménagères (résultats non publiés). Prolonger l'élevage au-delà de 8 semaines se traduit par une réduction pondérale des animaux, plus rapide sur boues urbaines que sur fumier de vache ou de cheval (NEUHAUSER et al., 1980). Cet effet dépressif dû à de multiples causes (appauvrissement nutritionnel, toxicité des fèces) serait donc moins marqué sur ordures ménagères et pourrait expliquer les très bons résultats 
TABLEAU 5

Influence d'un jeûne de $24 \mathrm{~h}$ sur la teneur en métaux lourds d'Eisenia fetida andrei. Composition des déjections obtenues pendant le jeûne et des déjections prélevées dans le milieu d'élevage (en $\mathrm{mg} / \mathrm{kg}$ de matière sèche).

Effect of discharge of excreta for $24 \mathrm{~h}$ on heavy metal content in Eisenia fetida andrei. Comparison between metal content of faeces excreted during discharge and faeces excreted on the growth medium (dry matter basis).

\begin{tabular}{|c|c|c|c|c|c|}
\hline & $\mathrm{Zn}$ & $\mathrm{Cd}$ & $\mathrm{Ni}$ & $\mathrm{Pb}$ & $\mathrm{Cr}$ \\
\hline $\begin{array}{r}\begin{array}{c}\text { Avant jeûne } \\
\text { Après jeûne }\end{array} \\
\text { Degré de signification de la différence }\end{array}$ & $\begin{array}{c}358 \\
163 \\
\text { T.H.S. }\end{array}$ & $\begin{array}{l}6,0 \\
5,1 \\
\text { N.S. }\end{array}$ & $\begin{array}{c}11,0 \\
7,1 \\
\text { T.H.S. }\end{array}$ & $\begin{array}{r}49,3 \\
9,3 \\
\text { T.H.S. }\end{array}$ & $\begin{array}{r}37,2 \\
6,2 \\
\text { T.H.S. }\end{array}$ \\
\hline Fèces et exsudats divers & 2126 & 8,4 & $(*)$ & 400 & $(*)$ \\
\hline Turricules & 2148 & 6,3 & 50,1 & 326 & 153 \\
\hline $\begin{array}{l}\text { Ordures ménagères } \\
\qquad \text { Degré de signification de la différence }\end{array}$ & $\begin{array}{l}1346 \\
\text { T.H.S. }\end{array}$ & $\begin{array}{l}8,6 \\
\text { N.S. }\end{array}$ & $\begin{array}{l}131 \\
\text { T.H.S. }\end{array}$ & $\begin{array}{l}243 \\
\text { N.S. }\end{array}$ & $\begin{array}{r}198 \\
\mathrm{~S} .\end{array}$ \\
\hline $\begin{array}{cr}\text { Indice }\left({ }^{* *}\right) & \text { Avant jeune } \\
\text { d'accumulation } & \text { Après jeune } \\
& \text { Degré de signification de la différence }\end{array}$ & $\begin{array}{l}0,29 \\
0,12 \\
\text { T.H.S. }\end{array}$ & $\begin{array}{l}0,96 \\
0,64 \\
\text { N.S. }\end{array}$ & $\begin{array}{r}0,08 \\
0,05 \\
\text { T.H.S. }\end{array}$ & $\begin{array}{r}0,22 \\
0,03 \\
\text { T.H.S. }\end{array}$ & $\begin{array}{r}0,23 \\
0,03 \\
\text { T.H.S. }\end{array}$ \\
\hline
\end{tabular}

(*) Pollution des échantillons lors du broyage.

$\left({ }^{* *}\right)$ Indice d'accumulation = concentration du métal dans E. fetida/concentration du métal dans le compost d'ordures ménagères.

enregistrés au cours de la présente expérience pour une période de 10 semaines. Les valeurs de 1,33 et 1,60 vers par cocon mis en élevage, qui englobent à la fois le taux d'éclosion et la mortalité post-natale, sont en accord avec celles citées dans la littérature (REINECKE \& KRIEL, 1981). Elles montrent que l'élevage d'E. fetida peut être conduit sur une période de 10 semaines sans entraîner de fortes mortalités. Les résultats sont conformes à ceux obtenus par ailleurs (FAYOLlE, 1982 ; de BLIGNÈRES, 1983) ; seule, APPELHOF (1981) enregistre une réduction de biomasse lombricienne après 3 mois d'élevage sur déchets ménagers.

Si l'on s'en tient à la définition stricte du concept d'accumulation donné par certains auteurs, on doit considérer que, dans le cas de cette expérience, seuls le zinc et le plomb donnent véritablement lieu à accumulation quand on passe d'un substrat qualifié à tort de référence (puisque non totalement dépourvu de métaux) à des ordures ménagères beaucoup plus polluées par des métaux. En fait, on doit admettre que ce sont les lombriciens élevés sur terreau témoin qui ont surtout donné lieu à accumulation, voire à concentration. La différence de comportement d' $E$. fetida élevé sur terreau de référence ou sur ordures ménagères peut être due à de multiples causes : le choix alimentaire fait par l'animal peut être différent en fonction de la nature du milieu d'élevage, ce qui entraîne des variations de la quantité totale de métal ingéré ; d'autre part, la biodisponibilité des métaux du milieu d'élevage (expliquée par la nature des liaisons contractées) est variable selon la constitution de ce dernier ; il est possible enfin que les lombriciens élevés dans un milieu à forte charge métallique développent un mécanisme de défense entraînant, par exemple, une éviction du plomb par augmentation de l'activité sécrétrice de l'organisme (WIELGUS-SERAFINSKA, 1979).

Cette observation souligne l'importance relative qu'il convient d'attribuer à la teneur totale d'un métal lourd donné d'un substrat lorsque ce dernier est des- tiné à servir de support à un élevage de vers; la nature des matières auxquelles le métal est lié et le type de liaison contractée paraissent jouer par contre un rôle beaucoup plus prépondérant. C'est ainsi que, d'après IRELAND (1983), Lumbricus rubellus Hoffmeister élevé sur sol contenant $4 \mathrm{ppm}$ de $\mathrm{Cd}$ concentre ce métal à $25 \mathrm{ppm}$, mais la concentration atteint $423 \mathrm{ppm}$ quand il est exposé à un milieu liquide contenant également $5 \mathrm{ppm}$ de cadmium. MORI \& KURIHARA (1979) observent par ailleurs qu' $E$. fetida, élevé sur un compost constitué d'un mélange de boues résiduaires urbaines et de sciures enrichi à $77 \mathrm{ppm}$ avec du cadmium, concentre ce métal jusqu'à $362 \mathrm{ppm}$.

L'expérience de jeûne réalisée au cours de ce travail permet également de mettre en évidence que l'enrichissement en métaux des vers élevés dans un milieu contaminé ne persiste pas systématiquement après élimination du contenu intestinal. Dans le cas de la présente expérience en effet, les indices d'accumulation de $\mathrm{Zn}$ et $\mathrm{Pb}$ sont fortement abaissés par rejet du métal dans les fèces ou élimination par voie épidermique (Wielgus-Serafinska \& Strzelec, 1983). Dans le cas du cadmium, l'indice d'accumulation est peu modifié par le jeûne : pour ce métal, il faut donc envisager, à la fois un transfert plus facile du contenu intestinal vers le corps de l'animal et une rétention plus énergique du métal absorbé, peut-être sous forme de complexes protéiniques de type métallo-thionine (SUZUKI et al., 1980 ; YAMAMURA et al., 1981).

Sur le plan pratique et dans le cas bien particulier d'un élevage sur ordures ménagères, il apparaît qu'une mise au jeûne d'E. fetida même de courte durée constitue un facteur d'amélioration important de la qualité, basée sur la richesse en certains métaux, du produit obtenu. Cette amélioration ne concerne cependant pas le cadmium, pour lequel il conviendrait de vérifier si une prolongation du jeûne ne suffirait pas à provoquer l'exclusion d'une partie du métal (TARRAdelas et al., 1982). Par ailleurs, la teneur en $\mathrm{Pb}$ se situe encore au niveau des teneurs maximales 
admissibles pour les aliments simples des animaux (10 ppm selon le J. O. du 16/12/1980). L’efficacité du jeûne pour l'amélioration de la qualité alimentaire des lombriciens issus d'autres milieux d'élevage que ceux constitués par des ordures ménagères demanderait à être confirmée.

\section{REMERCIEMENTS}

Ce travail a fait l'objet d'un contrat de recherche entre l'E.M.C. (Entreprise Minière et Chimique) et l'I.N.R.A. Nous remercions MM. EChard (E.M.C.) et de Beaucorps (S.C.P.A.) de l'intérêt qu'ils ont porté à la réalisation de ce programme.
Recu, le 27 juillet 1984.

Accepté le 6 mai 1985.

\section{RÉFÉRENCES BIBLIOGRAPHIQUES}

Appelhof M., 1981. Household scale vermicomposting. In «Workshop on the role of earthworms in the stabilization of organic re sidues ", Kalmazoo, Michigan Beech Leaf Press, 232-400.

Beyer W. N., 1980. Metals and terrestrial earthworms (Annelids : oligochaetal). In "Workshop on the role of earthworms in the stabilization of organic residues $"$, Kalamazoo, Michigan Beech Leaf Press, 137-150.

Blignères (de) F. X., 1983. Introduction expérimentale de vers dans un compost urbain. Compost Inf., 11, 11-19.

Bouché M. B., 1972. Lombriciens de France, Ecologie et systématique. Ann. Zool. Ecol. Anim., n ${ }^{\circ}$ spécial 72.

Bouché M. B., 1983. Les lombriciens et le traitement des déchets. Compost Inf., 11, 2-5.

Carmody F., 1979. Earthworm composting. A growing technology. Compost Sci., 20, 30-31.

Fayolle L., 1982. Etude de l'évolution du système déchets-lombriciens-micro-organismes : perspectives appliquées. Thèse DocteurIngénieur, Lyon, I, $130 \mathrm{p}$.

Franz M. F., 1978. The economic future of the earthworm in recycling. Compost Sci., 78, 23-26.

Gish C. D., Christensen R. E., 1973. Cadmium, nickel, lead and zinc in earthworms from roadside soil. Environ. Sci. Technol., 7, 1060-1062.

Graff O., 1974. Gewinnung von Biomasse aus Abfallstoffen durch Kultur des Kompostregenwurms Eisenia foetida (Savigny, 1826, Landbauforsch Völkenroode, 24, 137-142.

Hartenstein R., Neuhauser E. F., Collier J., 1980. Accumulation of heavy metals in the earthworm Eisenia fotida. J. Environ. Qual., 9, 23-26.

Hartenstein F., Hartenstein E., Hartenstein R., 1981. Gut load and transit time in the earthworm Eisenia foetida. Pedobiologia, 22, 5 20.

Helmke P. A., Robarge W. P., Korotev R. L., Schomberg P. J., 1979. Effects of soil-applied sewage sludge on concentration of elements in earthworms. J. Environ. Qual., 8, 322-327.

Hoenig M., Lima C., Dupire S., 1982. Validité des déterminations par spectrométrie d'absorption atomique avec atomisation électrothermique du cadmium, cobalt, chrome, nickel et plomb. Analusis, 10, $132-139$.

Ireland M. P., 1975. The effect of the earthworm Dendrobaena rubida on the solubility of lead, zinc and calcium in heavy metal contaminated soils in Wales. J. Soil Sci., 26, 313-318.

Ireland M. P., 1983. Heavy metal uptake and tissue distribution in earthworms. Earthworms and soil pullution, 247-265, in J. E. Satchell : «Earthworm Ecology », Chapman and Hall,
Juste C., Solda P., Dureau P., 1979. Etude des possibilités d'utilisation des composts d'ordures ménagères comme supports de culture maraîchère. Conv. Etude I.N.R.A.-Secr. Etat Environnement, $\mathrm{n}^{\circ} 75.136,54 \mathrm{p}$

Mitchell M. J., 1978. Role of invertebrates and micro-organisms in sludge decomposition. Proc. on « Utilization of soil organisms in sludge management », Syracuse, June 1978, Univ. New York Coll. and Envir. Sci and Forestry, 35-50.

Mitchell M. J., Hornor S. G., Abrams B. I., 1980. Decomposition of sewage sludge in drying beds and the potential role of the earthworms Eisenia foetida. J. environ. Qual., 9, 373-378.

Mori T., Kurihara Y., 1979. Accumulation of heavy metals in earthworms (Eisenia fotida) grown in composted sewage sludge. Sci. Rep. Tohoku Univ. Ser. IV (Biol.), 37, 289-297.

Neuhauser E. F., 1978. The utilization of earthworms in solid waste management. Proc. on "Utilization of soil organisms in sludge management », Syracuse, June 1978, Univ. New York ; Coll. and Envir. Sci and Forestry, 138-147.

Neuhauser E. F., Hartenstein F., Kaplan D. L., 1980. Growth of the earthworm Eisenia foetida in relation to population density and food rationing. Oikos, 35, 93-98.

Reinecke A. J., Kriel J. R., 1981. Influence of temperature on the reproduction of the earthworm Eisenia foetida (Oligochaeta). S Afr, J. Zool., 16 (2), 97-100.

Sabine J. R., 1980. Vermiculture as an option for resource recovery in the intensive animal industries. In "Workshop on the role of earthworms in the stabilization of organic residues ", Kalamazoo, Michigan, Beech Leaf Press, 243-252.

Suzuki K. T., Yamamura M., Mori T., 1980. Cadmium-binding proteins induced in earthworm. Arch. Environm. Contam. Toxicol., 9, 415-424.

Tarradelas J., Diercxsens P., Bouché M. B., 1982. Methods of extraction and analysis of PCBs of earthworms. Intern. J. Envir. Anal. Chem., 13, 55-67.

Wielgus-Serafinska E., 1979. Influence of lead poisoning and ultrastructural changes in the body wall of Eisenia foetida (Savigny), Oligochaeta. 1. Short action of different concentrations of lead and ultrastructural changes in the cells of the body wall. Folia Histochem. Cytochem., 17 (2), 181-188.

Wielgus-Serafinska E., Strzelec M., 1983. Influence of lead poisoning on ultrastructural changes in the body wall of Eisenia foetida (Savigny), Oligochaeta. II. Long action of different concentration of lead on ultrastructural changes in the cells on the body wall. Folia Histochem. Cytochem., 21 (2), 145-152.

Yamamura M., Mori T., Suzuki K. T., 1981. Metallothionein induced in the earthworm. Experientia, 37, 1187-1189. 